\title{
EXPORTACION DE SOLUTOS DESDE DIFERENTES USOS DEL SUELO. ESTUDIO EXPERIMENTAL EN EL PIRINEO CENTRAL ESPAÑOL
}

\author{
Teodoro LASANTA y José M. ${ }^{\mathrm{a}}$ GARCÍA-RUIZ
}

Instituto Pirenaico de Ecología, CSIC, Campus de Aula Dei, Zaragoza

\begin{abstract}
Resumen: En la Estación Experimental «Valle de Aísa» se han reproducido diferentes usos del suelo en parcelas cerradas, incluyendo cereales, prado, barbecho, agricultura cerealista itinerante, matorral denso y matorral quemado, entre otros. En este trabajo se analiza la concentración de solutos en la escorrentía procedente de cada una de las parcelas durante dos años (1996 y 1997). Los resultados muestran la gran influencia de las actividades humanas: las mayores pérdidas de nutrientes se producen en el barbecho, agricultura itinerante y cereal. Por el contrario, el matorral denso y el prado pierden pocos nutrientes, confirmando las moderadas exportaciones de agua y sedimentos en suspensión. La mayoría de los sólidos disueltos exportados son $\mathrm{HCO}_{3}^{-} \mathrm{y} \mathrm{Ca}^{2+}$. Otros nutrientes salen en cantidades muy pequeñas, en relación con el pobre contenido en nutrientes del suelo.
\end{abstract}

Palabras clave: Solutos, Sedimento en suspensión, Erosión del suelo, Usos del suelo, Pirineos.

Abstract: In the Aísa Valley Experimental Station nine different land-uses have been reproduced in closed plots, including cereal crop, meadows, fallow land, shifting agriculture, dense shrub cover and burnt shrub cover, among others. In this paper, solute concentration data from runoff yielded in each of the plots during two years (1996 and 1997) are analysed. The results show the dramatic influence of human activites: The highest losses of nutrients are yielded by the fallow land, shifting agiculture and cereal plots. In contrast, dense shurb cover and meadows lose few nutrients, confirming the moderate outputs of both runoff and suspended sediment. Most of the exported dissolved solids are $\mathrm{HCO}_{3}{ }^{-}$and $\mathrm{Ca}^{2+}$. Other nutrients are exported in very low quantities, in accordance with the poor nutrient content of the soil.

Key words: Solutes, Suspended sediment, Soil erosion, Land uses, Pyrenees. 


\section{INTRODUCCIÓN}

Es bien conocido el hecho de que los procesos de erosión solucionales pueden ser tan importantes como otros modos de erosión hídrica superficial (TRUDGILL, 1986). De hecho, la pérdida de nutrientes en suelos, cultivados o no, contribuye al descenso de la productividad, favoreciendo la pérdida de suelo y la aparición de otras señales de deterioro de los ecosistemas (véase, por ejemplo, SMALING y OENEMA, 1997). En la actualidad se dispone de información creciente sobre la concentración de solutos en aguas de escorrentía, pero el conocimiento es mucho más fragmentario sobre las principales fuentes de solutos y su distribución espacial (TRUDGILL, 1986).

Una gran variedad de estudios ha demostrado que las salidas de agua y sedimento se hallan frecuentemente relacionadas con las características de la cubierta vegetal y el tipo de uso del suelo (por ejemplo, EVANS, 1995; IMESON y VIS, 1984; KOSMAS et al., 1997; PAPY y BOIFFIN, 1989; RÜTTIMAN et al., 1995; LAL, 1996). La mayoría de los estudios concluyen que la intensidad de la erosión y la generación de escorrentía depende de la intensidad de las actividades humanas, y especialmente de la variabilidad espacial y temporal de la cubierta vegetal.

El estudio de las fuentes de solutos se enfrenta a muchas dificultades en áreas alteradas por el hombre (PIONKE y De WALLE, 1994), en gran parte debido al amplio rango de cubiertas vegetales y usos del suelo que pueden aparecer en una cuenca, y a la dificultad para observar directamente el origen y recorrido de los solutos. Una posible estrategia es reproducir diferentes usos del suelo en parcelas de una estación experimental y medir la escorrentía y las salidas de sedimento durante cada evento lluvioso, siendo constantes tanto el tipo de suelo como la pendiente y la exposición. Este procedimiento aporta información sobre la relativa importancia de solutos y sedimento en suspensión, así como los tipos de solutos exportados por cada uso del suelo. Si los usos del suelo están bien seleccionados, la información obtenida da una buena perspectiva sobre el origen de los solutos y su variabilidad temporal.

El principal objetivo de este trabajo es identificar los principales nutrientes exportados desde diferentes usos del suelo y comparar las pérdidas experimentadas por los sistemas tradicionales y actual de gestión del territorio. Un segundo objetivo consiste en situar las pérdidas de nutrientes en un contexto más amplio de erosión del suelo, en relación con la exportación de sedimento en suspensión.

\section{EL ÁREA DE ESTUDIO}

El estudio se ha llevado a cabo en el valle de Aísa (Figura 1), cuenca alta del río Aragón. Desde un punto de vista geológico, se trata de un área con una gran complejidad tectónica 


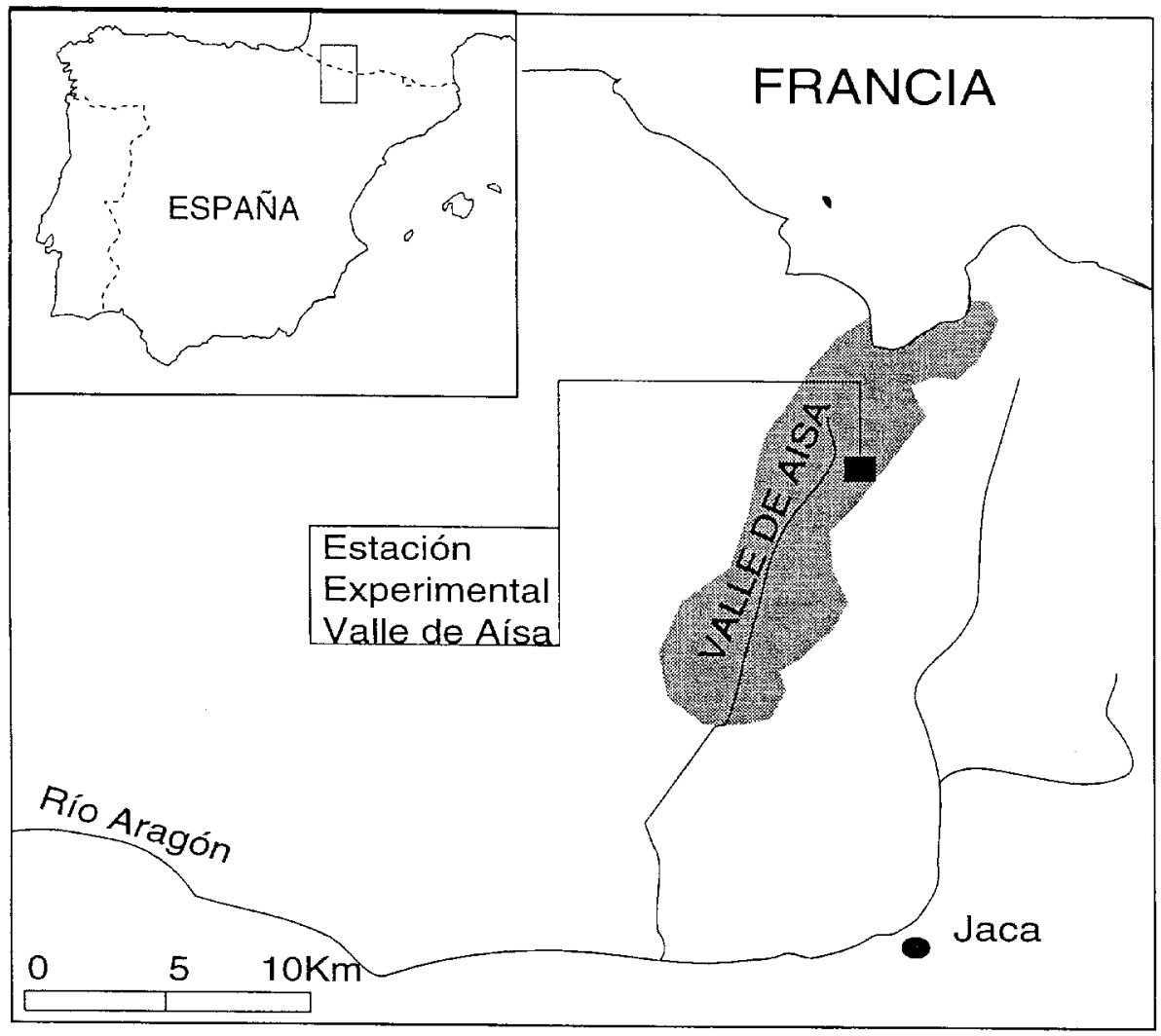

Figura 1. Localización de la Estación Experimental Valle de Aísa

y litológica. La parte superior del valle, ocupada por las Sierras Interiores, está compuesta por calizas y areniscas mesozoicas, formando un anticlinorio cabalgante hacia el sur, con. abruptos escarpes, circos glaciares, canales de aludes y superficies intensamente karstificadas. Más al sur, el sector de flysch eoceno ocupa la mayor parte del valle, con divisorias redondeadas y vertientes regularizadas, sobre las que destacan ocasionalmente cordones morrénicos laterales y depósitos glacio-lacustres colgados.

Temperatura y precipitación muestran un fuerte gradiente de norte a sur, relacionado con el descenso altimétrico y con el juego de influencias atlánticas y mediterráneas. En las áreas más elevadas se ha estimado una precipitación media anual superior a $2.000 \mathrm{~mm}$ (RIJCKBORST, 1967), mientras la localidad de Aísa, a 1.100 m s.n.m. recibe una precipitación media de $1.100 \mathrm{~mm}$, con una temperatura media anual de $10^{\circ} \mathrm{C}$. La isoterma de $0^{\circ} \mathrm{C}$ durante la estación fría (noviembre-abril) se localiza a $1.650 \mathrm{~m}$ (GARCIA RUIZ et al., 1986).

Por debajo de $1500 \mathrm{~m}$ los bosques de Pinus sylvestris dominan en las umbrías, mientras en las solanas quedan bosquetes de Quercus gr. faginea alternando con matorrales submediterráneos (Buxus semperoirens, Genista scorpius, Echinospartum horridum, Rosa gr. canina y 
Junipresus communis). Por encima de $1.500 \mathrm{~m}$ los bosques densos de Pinus sylvestris ocupan tanto las laderas solanas como las umbrías, con manchas de Fagus sylvatica y Abies alba. El piso subalpino (por encima de 1700-1800 m) fue casi completamente deforestado desde el siglo XI con el fin de aumentar la extensión de los pastos de aprovechamiento estival.

Durante siglos, los cereales fueron el cultivo más importante. Los prados, en cambio, tuvieron un papel muy limitado. En los periodos de mayor presión demográfica (por ej., a mediados del siglo XIX) muchas laderas pendientes en exposición solana fueron también cultivadas mediante sistemas itinerantes, utilizando cenizas como abono (articas) y con escasas técnicas de conservación del suelo. En promedio, en el Pirineo Central español se cultivó casi el $30 \%$ de la superficie situada por debajo de $1.600 \mathrm{~m}$ (LASANTA, 1989). Las laderas cubiertas de matorral fueron quemadas frecuentemente con el fin de favorecer el desarrollo temporal de pastos.

Durante el siglo xx el cambio más destacado en el uso del suelo ha sido la reducción de la superficie cultivada. Las laderas pendientes han sido totalmente abandonadas, de manera que el área de cultivos se reduce a los fondos de valle, conos de deyección y rellanos colgados con depósitos glaciolacustres, todos ellos con pendientes suaves y suelos profundos, localizados cerca de los pueblos (LASANTA, 1988). Por otro lado, los campos de cereal han sido sustituidos por prados. Los campos abandonados han sido colonizados por comunidades herbáceas y por matorral (MOLINILLO et al., 1997).

\section{MÉTODOS}

La mayor parte de la información que se presenta en este trabajo procede de la Estación Experimental Valle de Aísa, localizada a $1200 \mathrm{~m}$ s.n.m., sobre una parcela que fue abandonada hace 35 años y que al inicio del estudio estaba completamente cubierta por un matorral denso de Genista scorpius y Rosa gr. canina. Inicialmente se instalaron 6 parcelas cerradas de $10 \times 3 \mathrm{~m}$, incluyendo en su parte final una trampa Gerlach desde donde el agua de escorrentía superficial se dirige hacia unos balancines conectados con data loggers, con el fin de registrar continuamente la generación de escorrentía. Se dispone también de un pluviómetro conectado a un data logger. Parte de la escorrentía es finalmente desviada hacia depósitos de plástico, vaciados después de cada evento lluvioso para analizar la concentración de sedimento en suspensión y de solutos, así como la composición de estos últimos.

Las primeras 6 parcelas, instaladas en 1991, reproducen diferentes usos del suelo (Figura 2):

- Cereal (cebada) fertilizado con cenizas, siguiendo un procedimiento similar al cultivo itinerante (artica). 


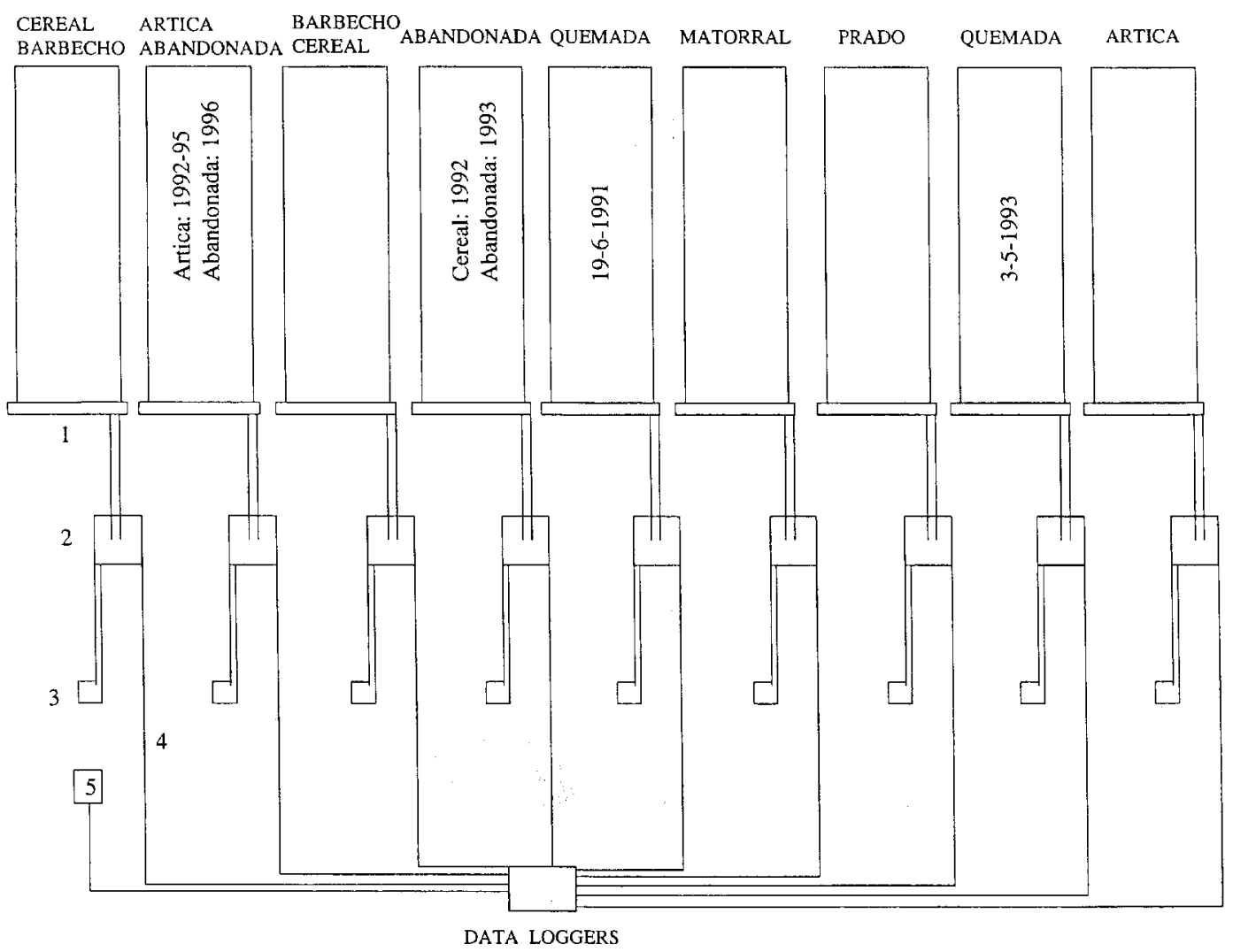

1 Canal Gerlach

2 Dispositivo de canjilones

3 Colector de sedimentos

4 Cables de conexión

5 Pluviografo

Figura 2. La Estación Experimental Valle de Aísa.

Distribución de las parcelas experimentales y su evolución

- Barbecho: parcela no cultivada, pero si labrada.

- Matorral quemado.

- Matorral denso con la vegetación original del campo abandonado sobre el que se instaló la Estación Experimental.

- Prado. 
En 1993 la parcela de barbecho pasó a cultivarse con cereal, mientras la parcela original de cereal se convirtió en rastrojo después de la cosecha, iniciando un proceso de abandono puesto que a partir de entonces no recibió ningún otro tipo de manipulación. Además en 1993 se añadieron 2 nuevas parcelas:

- Barbecho, que posteriormente fue cultivada con cereal, alternando un año tras otro con barbecho.

- Nueva parcela de matorral quemado, con el fin de confirmar las tendencias observadas en la parcela incendiada en 1991.

Todavía en 1996 se instaló una nueva parcela de artica, mientras que la primera fué dejada como campo abandonado.

Los análisis de contenido iónico de las aguas de escorrentía $\left(\mathrm{Na}^{+}, \mathrm{K}^{+}, \mathrm{Ca}^{2+}, \mathrm{Mg}^{2+}, \mathrm{HCO}_{3}^{-}\right.$, $\mathrm{Cl}, \mathrm{SO}_{4}{ }^{2-}, \mathrm{NO}_{3}{ }^{-}, \mathrm{SiO}_{2}$ ) se llevaron a cabo mediante espectroscopia de absorción atómica en el caso de los cationes y mediante volumetría y colorimetría en el caso de los aniones.

Las nueve parceles actuales cubren varias posibilidades de usos del suelo, incluso los correspondientes al llamado sistema tradicional (cereal, artica, matorral quemado, barbecho), tanto en los Pirineos como en gran parte de las montañas mediterráneas.

Es importante tener en cuenta que el empleo de parcelas experimentales para estimar la escorrentía y la exportación de sedimento en suspensión y de solutos presenta muchas limitaciones. La característica de las parcelas (de tamaño relativamente pequeño) y la perturbación causada en el suelo por su instalación reduce la validez absoluta de los datos, pero los resultados son buenos indicadores de las diferencias relativas entre los usos del suelo. De hecho, las medidas en parcelas experimentales sólo son aceptadas para propósitos comparativos, es decir, para proporcionar órdenes de magnitud de la escorrentía y la erosión en diferentes condiciones ambientales (LÓPEZ BERMÚDEZ et al., 1993).

Finalmente, se ha manejado información sobre el contenido de solutos del agua de lluvia en una localidad próxima (San Juan de la Peña), en el Pirineo Central español (ALVERA, 1990), con el fin de comparar sus valores con los obtenidos en las parcelas experimentales.

En el valle de Aísa se tomaron 79 y 60 muestras de suelo de campos abandonados y campos cultivados respectivamente. Para cada una de ellas se realizaron análisis físico-químicos, incluyendo granulometría y contenido en Ca, materia orgánica, N, P, K y Mg. Tales análisis se llevaron a cabo en el Laboratorio de Análisis Agrarios de la Comunidad de La Rioja.

Es importante tener en cuenta que en la parcela cultivada con cereal se añadió anualmente fertilizante químico, con $\mathrm{NH}_{4}, \mathrm{P}_{2} \mathrm{O}_{5}$ y $\mathrm{K}_{2} \mathrm{O}$ como principales componentes, $\mathrm{y}$ pequeñas cantidades de $\mathrm{Mg}$, S y Fe. Obviamente, las parceles en barbecho y abandonada 
(previamente cultivada con cereal) han recibido (uno o varios años antes) cantidades similares de abono químico.

\section{RESULTADOS}

\section{Características principales de los suelos}

La distribución granulométrica indica que la mayor parte de los muestras de suelo son francas o franco-arcillosas, de acuerdo con la clasificación de la USDA, 1951. En promedio, el $38 \%$ es arena, el $36 \%$ limo y el $25 \%$ arcilla (RUIZ FLAÑO, 1993; LASANTA, 1989). Los suelos son poco potentes (en general con menos de $30 \mathrm{~cm}$ de profundidad), pedregosos (entre 30 y $60 \%$ en superficie, llegando en muchos casos al $100 \%$ ), y con un alto contenido en carbonatos $(32.7 \%)$, como corresponde a suelos desarrollados sobre un sustrato carbonatado. Se han descrito como regosoles, con estructura subangular o angular (PARDINI et al., 1991).

Los análisis químicos demuestran que los suelos del área de estudio, tanto los cultivados como los abandonados, tienen bajos contenidos en $\mathrm{P}, \mathrm{K}$ y $\mathrm{Mg}$ y poseen abundante $\mathrm{N}$. El contenido de materia orgánica es relativamente alto (entre el 1 y el 2\% en el $50 \%$ de las muestras, mientras que el $45 \%$ superan ese umbral), lo que explica los elevados niveles de $\mathrm{N}$.

El contenido en P está entre niveles bajos y muy bajos, especialmente en los campos abandonados, donde el $81 \%$ de las muestras no alcanzan 6 ppm, y el 16\% de las muestras está entre 6 y 12 ppm. Estos datos se deben probablemente a procesos de erosión difusa, ya que los fosfatos se hallan fuertemente ligados a las partículas del suelo y son arrastradas con ellas por la escorrentía superficial. La Tabla 1 indica que las muestras de suelo tomadas de la parte baja de los campos presentan un mayor contenido en P que las tomadas de la parte superior, demostranđo una redistribución espacial relacionada con la escorrentía (RUIZ FLAÑO, 1993).

Tabla 1. Análisis de varianza entre el contenido en fósforo (ppm) y la localización de las muestras dentro de cada campo. $\mathrm{N}=79$

\begin{tabular}{|lcrcc|}
\hline Localización & Número & Media & Desv. stand. & Error stand. \\
\hline Parte superior & 25 & 3,16 & 2,05 &, 41 \\
Parte media & 28 & 3,44 & 1,98 &, 37 \\
Parte inferior & 26 & 5,11 & 3,24 &, 63 \\
\hline
\end{tabular}

F-Test:p $=.0225$

Fuente: Ruiz-Flaño (1993) 
En el caso de $\mathrm{K}$, el $43 \%$ de las muestras presenta contenidos muy bajos (menos de 0.20 meq/100), y el $42 \%$, contenidos bajos (entre 0.21 y $0.40 \mathrm{meq} / 100$ ), correspondiendo los mejores resultados a los campos abandonados. Por lo que respecta a $\mathrm{Mg}$, el contenido es también muy deficiente, siempre por debajo de 1 meq/100.

Finalmente, la Tabla 2 incluye información sobre el contenido de nutrientes de Genista scorpius, a partir del análisis de la materia seca. Genista scorpius es la especie dominante entre las formaciones de matorral y fue habitualmente quemada para distribuir las cenizas en los campos afectados por sistemas de agricultura itinerante. Los resultados muestran que Gestina scorpius aporta cantidades extremadamente bajas de nutrientes (GARCIA RUIZ

Tabla 2. Contenido de nutrientes en Genista scorpius (resultados sobre materia seca).

\begin{tabular}{|lr|}
\hline Nitrógeno & $1,24 \%$ \\
Fósforo & $0,05 \%$ \\
Polasio & $0,29 \%$ \\
Calcio & $0,53 \%$ \\
Magnesio & $0,08 \%$ \\
Sodio & $33 \mathrm{ppm}$. \\
\hline
\end{tabular}

et al., 1996). Las cenizas sólo representan el $2.25 \%$ del peso de la planta y las proporciones de $\mathrm{P}, \mathrm{Mg}$ y $\mathrm{N}$ son insignificantes. Este análisis demuestra que la quema de matorral no enriquece mucho el suelo y ello explica el que la productividad de las articas fuera muy baja, a la vez que la colonización vegetal, una vez abandonado el campo, fuera muy lenta.

\section{La pérdida de solutos en diferentes usos del suelo}

El período de estudio incluye los años 1996 y 1997. El año 1996 puede considerarse húmedo, con una precipitación total de $1.389 \mathrm{~mm}$, mientras que la precipitación de 1997 estuvo muy próxima a la media, con $1.140 \mathrm{~mm}$. La Figura 3 muestra la distribución mensual de la precipitación y el extremo periodo húmedo a finales de 1996. Así, noviembre de 1996 fue muy húmedo (246 mm), seguido por un mes de diciembre aún más húmedo (275 $\mathrm{mm}$ ). En 1997, noviembre fue muy lluvioso de nuevo (266 mm), pero, por el contrario, diciembre fue muy seco. Los valores de primavera fueron casi normales, aunque en junio de 1996 fue más seco que la media, y mayo de 1997 más húmedo.

La importancia relativa de las pérdidas de solutos y sedimento en suspensión en cada uso del suelo se incluye en las Tablas 3 (1996) y 4 (1997). Los resultados muestran que existe mayor variabilidad en el caso de la concentración de sedimento en suspensión que 


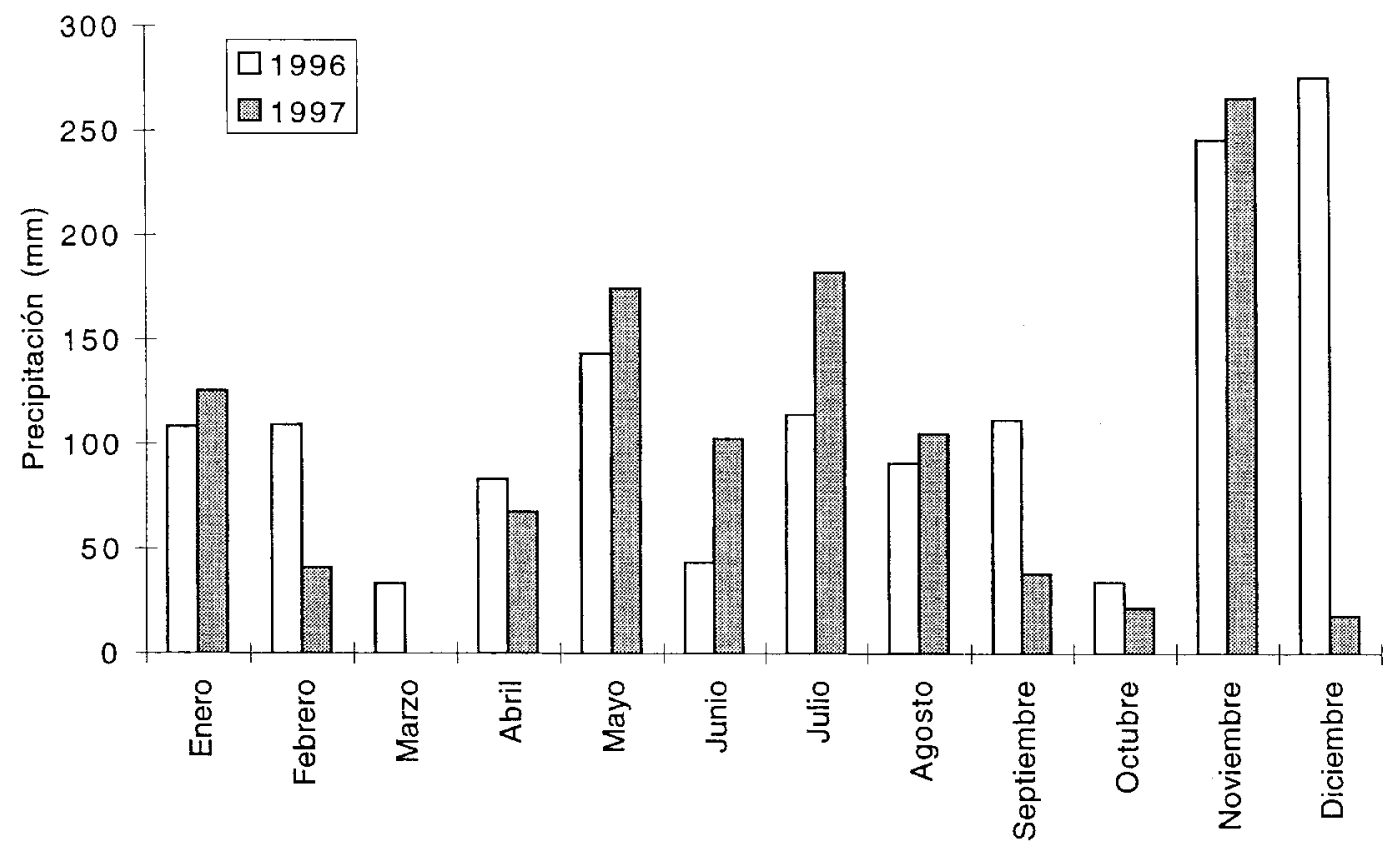

Figura 3. Distribución mensual de la precipitación en 1996 y 1997 en la Estación Experimental Valle de Aísa

Tabla 3. Escorrentía, solutos y sedimento en suspensión en diferentes usos del suelo (1996)

\begin{tabular}{|c|c|c|c|c|c|c|c|}
\hline & \multirow{2}{*}{$\begin{array}{c}\text { Coef. Escorr. } \\
(\%)\end{array}$} & \multicolumn{2}{|c|}{ Concentr. de sedimento (mg..$^{-1}$ ) } & \multicolumn{2}{|c|}{ Pérdidas de suelo $\left(g \cdot m^{-2}\right)$} & \multirow[b]{2}{*}{ Total } & \multirow{2}{*}{$\begin{array}{c}\text { Porcentaje } \\
\text { de soluto }\end{array}$} \\
\hline & & Solutos & Sed. en Susp. & solutos & Sed. en Susp & & \\
\hline Matorral denso & 3,9 & 162 & 62 & 9,0 & 3,4 & 12,4 & 72,5 \\
\hline Pasto & 5,6 & 145 & 113 & 11,2 & 8,7 & 19,9 & 56,3 \\
\hline Parcela quemada 1 & 4,1 & 152 & 34 & 8,8 & 2,0 & 10,8 & 81,5 \\
\hline Parcela quemada 2 & 5,5 & 156 & 611 & 11,9 & 46,5 & 58,4 & 20,3 \\
\hline Cereal abandonado & 10,1 & 164 & 38 & 23,0 & 5,3 & 28,3 & 81,3 \\
\hline Cereal & 9,2 & 186 & 712 & 23,9 & 91,4 & 115,3 & 20,7 \\
\hline Barbecho & 7,7 & 180 & 158 & 13,9 & 12,2 & 26,1 & 53,3 \\
\hline Artica & 9,4 & 202 & 297 & 18,3 & 26,9 & 45,2 & 40,5 \\
\hline Artica abandonada ${ }^{*}$ & 6,5 & 175 & 524 & 19,3 & 57,6 & 76,9 & 25,1 \\
\hline
\end{tabular}

* Sólo durante 10 meses (Marzo-Diciembre, 1996).

de solutos. Esta diferencia se explica porque los solutos están siempre presentes en la escorrentía, incluso con caudales muy bajos, mientras que la concentración de sedimento en suspensión varía mucho con el caudal. Sin embargo, a pesar de la relativa regularidad de los solutos, pueden distinguirse tres grupos de usos del suelo, con escasa diferencia de comportamiento en los dos años estudiados: 
Tabla 4. Escorrentía, solutos y sedimento en suspensión en diferentes usos del suelo (1997)

\begin{tabular}{|c|c|c|c|c|c|c|c|}
\hline \multirow[b]{3}{*}{ Matorral denso } & \multirow{2}{*}{$\begin{array}{c}\text { Coef. Escorr. } \\
(\%)\end{array}$} & \multicolumn{2}{|c|}{ Concentr de sedimento $\left(m g \cdot l^{-1}\right)$} & \multicolumn{2}{|c|}{ Pérdidas de suelo(g.m $\left.m^{2}\right)$} & \multirow{2}{*}{ Total } & \multirow{2}{*}{$\begin{array}{l}\text { Porcentaje. } \\
\text { de soluto }\end{array}$} \\
\hline & & Solutos & Sed.en Susp. & solutos $\quad S$ & Sed.en Susp & & \\
\hline & 5,8 & 130 & 37 & 8,6 & 2,5 & 11,1 & 77,5 \\
\hline Pasto & 6,9 & 124 & 33 & 9,8 & 2,6 & 12,4 & 79,0 \\
\hline Parcela quemada 1 & 5,9 & 123 & 23 & 8,3 & 1,5 & 9,8 & 84,7 \\
\hline Parcela quemada 2 & 8,6 & 140 & 292 & 13,7 & 28,5 & 42,2 & 32,5 \\
\hline Cereal abandonado & 9,5 & 169 & 77 & 18,3 & 8,3 & 26,6 & 68,8 \\
\hline Cereal & 8,4 & 314 & 50 & 30,0 & 4,7 & 34,7 & 86,5 \\
\hline Barbecho & 12,7 & 178 & 326 & 25,7 & 47,2 & 72,9 & 35,3 \\
\hline Artica & 13,7 & 198 & 416 & 27,9 & 58,6 & 86,5 & 32,3 \\
\hline Artica abandonada & 12,3 & 167 & 291 & 26,1 & 45,4 & 71,5 & 36,5 \\
\hline
\end{tabular}

- Los valores más bajos de concentración de solutos se registran en la parcela de prado, las dos parcelas quemadas y la parcela de matorral denso, es decir, allí donde los trabajos agrícolas están ausentes y donde se ha alcanzado un buen recubrimiento vegetal (incluso algunos años después de un incendio).

- Los valores medios se obtienen en la parcela de cereal abandonado y en la artica abandonada, es decir, en las parcelas que fueron labradas y cultivadas unos años antes, y que ahora están sujetas a un estadio inicial de colonización vegetal. Estas parcelas fueron fertilizadas mientras se cultivaron (con cenizas en el caso de la artica) y por ello el nivel de concentración de solutos es moderadamente alto.

- Los mayores valores se registran en la parcela de cereal, el barbecho y en la artica activa, donde se produce una removilización anual del suelo y se añaden fertilizantes químicos o cenizas.

Así, para la concentración de solutos la clasificación de los usos del suelo va desde las parcelas menos perturbadas a aquellas en las que el cultivo está funcionando plenamente.

Por lo que respecta a la pérdida total de solutos (en $\mathrm{g} \mathrm{m}^{-2}$ ), la parcela quemada en 1993 (ya en 1996 muy bien recolonizada por el matorral) y la parcela de matorral denso registran los valores más bajos, seguidos por la parcela de prado y la parcela quemada en 1996. El resto de las parcelas muestran un comportamiento más irregular, en gran parte relacionado con el coeficiente de escorrentía. No obstante, interesa destacar que la parcela de cereal es, en los dos años estudiados, el uso que produce las pérdidas más elevadas de solutos (23.9 $\mathrm{g}$ m-2 en 1996 y $30 \mathrm{~g} \mathrm{~m}$-2 en 1997). De esta forma, en el caso de la exportación de solutos los usos del suelo pueden clasificarse en dos grupos, incluyendo en el primero las parcelas sin perturbación o con densa cubierta vegetal, y en el segundo las parcelas afectadas por laboreo y cultivo o que han sido abandonadas recientemente. La razón que explica la inclusión de las parcelas abandonadas en este segundo grupo, a pesar de que por concentración de solutos formaban un grupo intermedio con valores moderados, es que presentan elevados coeficientes de escorrentía, incrementando así la exportación total de materiales. 
Si la pérdida de suelo se estudia globalmente (Figura 4), en ese caso la proporción de solutos en relación con la pérdida total de sedimento demuestra que algunas parcelas con valores bajos de erosión de suelo presentan una mayor pérdida de solutos que de sedimento en suspensión. Por el contrario, las parcelas con elevados valores de pérdida de suelo registran una mayor salida de sedimento en suspensión. Es decir, los materiales particulados contribuyen mucho a la exportación total de sedimento. Estos resultados significan que algunos usos del suelo (matorral denso, parcelas quemadas, prados y, en menor medida, la parcela de cereal abandonado) producen pocos solutos y aun menos sedimento en suspensión. El resto de los usos producen relativamente altas cantidades de solutos y aún más sedimento en suspensión, dando lugar a valores elevados de pérdida total de suelo. La parcela cultivada con cereal se comporta de manera sorprendente, con cifras muy bajas de sedimento en suspensión en 1997, debido probablemente a errores no definidos en el sistema de medición.

Las Tablas 5 (1996) y 6 (1997) se refieren a las pérdidas de los solutos más representativos en cada uno de los usos del suelo, en mg m-2. Los resultados obtenidos para el fósforo no se incluyen, ya que los valores están próximos a cero como consecuencia de los niveles muy bajos de este elemento en el suelo.

Los salidas más significativas de solutos están en forma de $\mathrm{HCO}_{3}^{-}$, con pérdidas próximas a $20 \mathrm{~g} \mathrm{~m}^{-2}$ año-1 en la artica activa y abandonada, así como en la parcela de cereal. Evidentemente, $\mathrm{Ca}^{2+}$ se exporta también en cantidades altas (alrededor de $5 \mathrm{~g} \mathrm{~m}^{-2}$ año ${ }^{-1}$ en las parcelas citadas), mientras que el resto de los solutos muestran valores muy bajos, especialmente $\mathrm{Na}^{+}$y $\mathrm{Mg}^{2+}$ (debido a su bajo contenido en el suelo), $\mathrm{SO}_{4}^{-2}$ y $\mathrm{NO}_{3}^{-}$. En el caso de $\mathrm{SiO}_{2}$ y $\mathrm{K}^{+}$los valores obtenidos pueden considerarse moderados.

Es importante tener en cuanta que en los usos del suelo con salidas relativamente bajas de solutos (por ej., matorral denso, prado y parcelas quemadas), $\mathrm{HCO}_{3}^{-} \mathrm{y} \mathrm{Ca}^{2+}$ representan más del $80 \%$ del total de solutos exportados, mientras en el resto de los usos esta cifra desciende hasta el $60 \%$ incluso menos, ya que estas parcelas también exportan cantidades relativamente altas de otros solutos. Si el comportamiento de cada soluto se estudia para cada uso del suelo, entonces deben resaltarse los siguientes resultados:

- $\mathrm{K}^{+}$se exporta sobre todo en la parcela de barbecho, seguida por la parcela de cereal, el cereal abandonado y la artica.

- $\mathrm{SiO}_{2}$ se exporta en cantidades relativamente altas en la artica y en el cereal abandonado.

- $\mathrm{Cl}^{-}$sale sobre todo de la parcela de cereal.

- $\mathrm{NO}_{3}{ }^{-}$se exporta principalmente en la parcela de barbecho y de cereal.

- $\mathrm{Mg}^{2+}$ sale en pequeñas cantidades de todas las parcelas, pero las cifras son ligeramente superiores en la de cereal. 


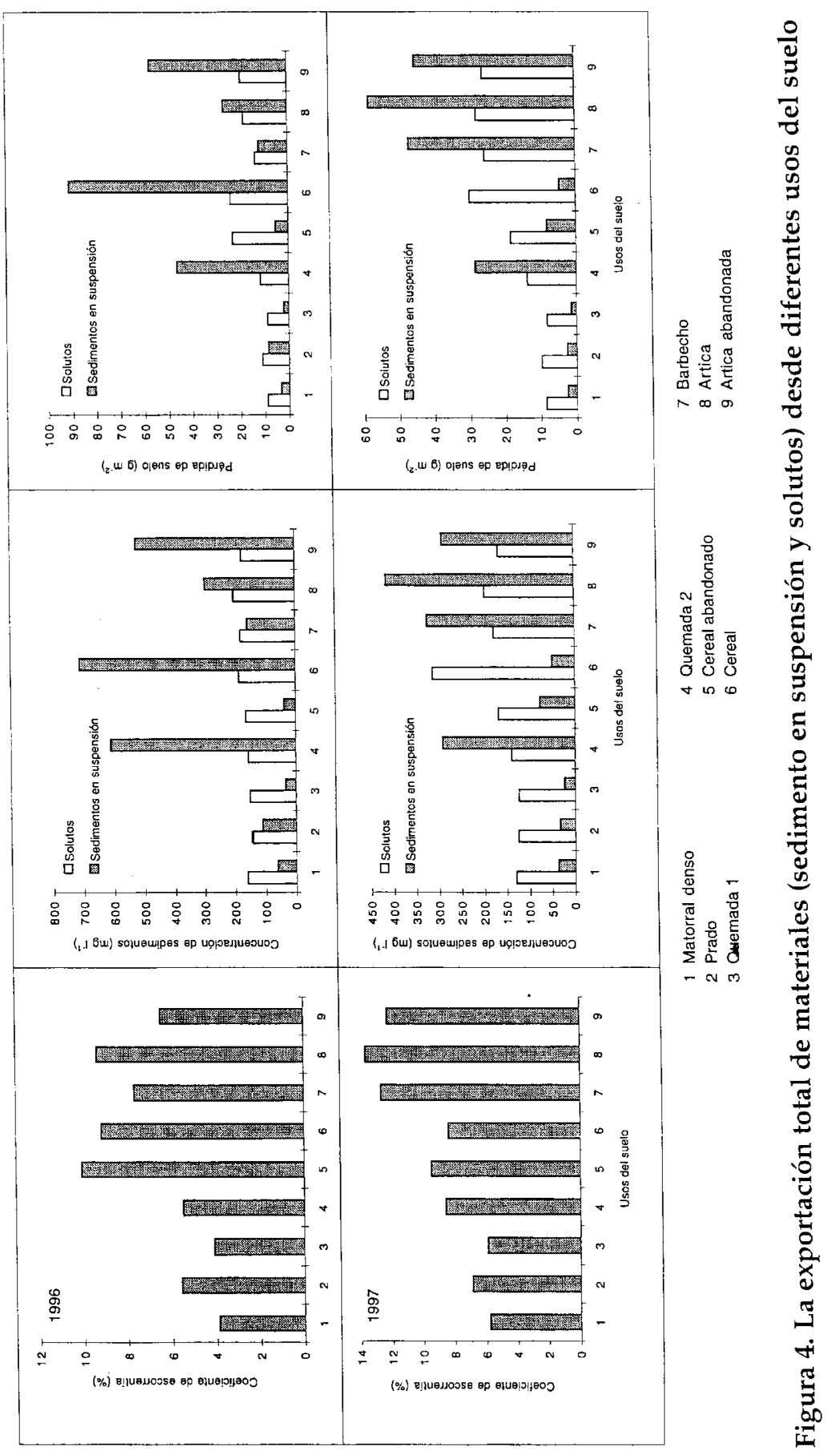


Tabla 5. Solute output from different land-uses (mg.m-2) A'sa Valley Experimental Station, 1996

\begin{tabular}{|lccccccccc|}
\hline Uso del suelo & $\mathrm{Ca}^{2+}$ & $\mathrm{Mg}^{2+}$ & $\mathrm{Na}^{+}$ & $\mathrm{K}^{+}$ & $\mathrm{SO}_{4}^{-}$ & $\mathrm{SiO}_{2}$ & $\mathrm{Cl}^{-}$ & $\mathrm{NO}_{3}^{-}$ & $\mathrm{HCO}_{3}{ }^{-}$ \\
\hline Cereal abandonado & 5.570 & 232 & 106 & 614 & 59 & 761 & 186 & 82 & 17.397 \\
Barbecho & 3.167 & 80 & 82 & 313 & 170 & 382 & 240 & 97 & 9.626 \\
Parcela quemada 1 & 1.817 & 71 & 40 & 222 & 23 & 292 & 73 & 49 & 5.990 \\
Parcela quemada 2 & 2.412 & 84 & 58 & 252 & 137 & 277 & 140 & 86 & 8.723 \\
Prado & 2.397 & 80 & 45 & 364 & 16 & 399 & 182 & 59 & 7.748 \\
Matorral denso & 1.503 & 73 & 30 & 198 & 67 & 296 & 84 & 27 & 5.148 \\
Cereal & 5.568 & 276 & 127 & 365 & 796 & 432 & 515 & 268 & 18.044 \\
Artica & 3.753 & 195 & 101 & 360 & 168 & 678 & 244 & 50 & 12.249 \\
Artica abandonada(*) & 4.256 & 224 & 159 & 279 & 528 & 432 & 142 & 85 & 14.134 \\
\hline
\end{tabular}

*Only during 10 months (March-December, 1996)

Tabla 6. Solute output from different land-uses (mg.m-2) A'sa Valley Experimental Station, 1997

\begin{tabular}{|lccccccccc|}
\hline Uso del suelo & $\mathrm{Ca}^{2+}$ & $\mathrm{Mg}^{2+}$ & $\mathrm{Na}^{+}$ & $\mathrm{K}^{+}$ & $\mathrm{SO}_{4}^{-}$ & $\mathrm{SiO}_{2}$ & $\mathrm{Cl}^{-}$ & $\mathrm{NO}_{3}^{-}$ & $\mathrm{HCO}_{3}^{-}$ \\
\hline Cereal abandonado & 3.683 & 126 & 62 & 542 & 23 & 327 & 202 & 71 & 11.956 \\
Barbecho & 4.788 & 145 & 178 & 766 & $*$ & 370 & $*$ & 406 & 12.255 \\
Parcela quemada 1 & 2.161 & 92 & 51 & 330 & 126 & 308 & 120 & 91 & 7.314 \\
Parcela quemada 2 & 2.992 & 114 & 93 & 346 & 97 & 389 & 167 & 108 & 11.383 \\
Prado & 2.461 & 81 & 62 & 317 & 20 & 418 & 116 & 68 & 8.388 \\
Matorral seco & 2.089 & 75 & 34 & 339 & 7 & 397 & 105 & 34 & 7.216 \\
Cereal & 5.061 & 304 & 139 & 527 & $*$ & 418 & 404 & 162 & 17.647 \\
Artica & 5.311 & 189 & 113 & 506 & 126 & 647 & 279 & 158 & 18.489 \\
Artica abandonada(*) & 5.520 & 279 & 146 & 400 & $*$ & 531 & 262 & 46 & 19.734 \\
\hline
\end{tabular}

* Unreliable data

El contenido de solutos en el agua de lluvia de San Juan de la Peña (Alvera, 1990) aporta información muy interesante a efectos comparativos. Ca2+ es el elemento más abundante en el agua de lluvia (alrededor de $1,23 \mathrm{mg} \mathrm{l-1)}$, seguido por $\mathrm{N}(0,632 \mathrm{mg} \mathrm{l-1}), \mathrm{Mg} 2+(0,328$ mg l-1) y $\mathrm{Na}+(0,16 \mathrm{mg} 1-1)$, mientras $\mathrm{K}+$ y P están presentes en cantidades muy bajas. Si se aceptan estos contenidos de solutos para la precipitación del valle de Aísa, entonces en 1996 y 1997 la entrada de solutos oscila alrededor de los siguientes valores: entre 1400 y $1700 \mathrm{mg}$ $\mathrm{m}-2$ para $\mathrm{Ca} 2+$; entre 700 y $900 \mathrm{mg} \mathrm{m}-2$ para $\mathrm{N}$; entre 350 y $450 \mathrm{mg} \mathrm{m}-2$ para $\mathrm{Mg} 2+$; entre 190 y $250 \mathrm{mg} \mathrm{m}-2$ para $\mathrm{Na}+$; entre 40 y $60 \mathrm{mg} \mathrm{m-2}$ para $\mathrm{K}+$; y entre 15 y $25 \mathrm{mg} \mathrm{m}-2$ para $\mathrm{P}$. 


\section{DISCUSIÓN Y CONCLUSIONES}

Los suelos desarrollados sobre sustrato flysch en el Pirineo español son muy pobres en nutrientes, especialmente si han sido cultivados durante siglos. Los contenidos en P, K y Mg están clasificados en la mayor parte de las muestras como bajos o muy bajos, mientras que la presencia de $\mathrm{N}$ puede considerarse como abundante. El cultivo ha dado lugar a una redistribución de los nutrientes en las laderas y por ello es por lo que los valores son claramente más elevados en la parte baja de los campos que en la parte alta. Probablemente, las características del suelo explican la casi exclusiva presencia de Genista scorpius durante décadas después del abandono del cultivo, ya que este matorral presenta bajos niveles de exigencia de nutrientes. Este hecho también contribuye a la explicación de la escasa productividad del cereal en la agricultura nómada, que estuvo ampliamente extendida en las montañas mediterráneas. Esta es la razón por la que algunos autores identifican a la agricultura nómada como la principal causa de erosión del suelo en muchas laderas (GARCÍA RUIZ et al., 1995).

Los estudios llevados a cabo en la Estación Experimental Valle de Aísa confirman que los uso del suelo influyen en gran medida sobre la exportación de solutos, en parte porque también controlan la escorrentía superficial. SWANK (1986) señala que un factor muy importante en el incremento de la salida de solutos es el aumento de escorrentía que resulta de cualquier perturbación de la cubierta vegetal densa, natural o seminatural. Los resultado obtenidos demuestran que hay diferencias muy significativas entre las parcelas experimentales afectadas por escasa perturbación y aquellas influidas actualmente por actividades humanas. Así, en el primer caso, el matorral denso, el prado y las parcelas quemadas algunos años antes (y actualmente recolonizadas con matorral) registran valores más bajos de concentración y exportación de solutos. Por el contrario, la agricultura nómada (articas), el cultivo de cereal y el barbecho producen grandes cantidades de solutos. En una posición intermedia, los campos abandonados confirman que el abandono de tierras de cultivo representa un descenso en la exportación de solutos (como también de sedimento en suspensión).

Las relaciones existentes entre la proporción de solutos y de sedimento en suspensión están de acuerdo con otros estudios a escala de cuencas. Así, en cuencas forestales escasamente perturbadas donde, en general, la pérdida total de sedimento es pequeña, la salida de solutos es mayor que la de sedimento en suspensión (por ej., FELLER y KIMMINS, 1981; VERSTRATEN, 1997; PUIGDEFÁBREGAS y ALVERA, 1986). LLORENS et al. (1997) han registrado una elevada y sostenida concentración de sólidos disueltos y una baja y muy variable concentración de sedimento en suspensión, en una cuenca de terrazas abandonadas desde hace décadas en el Pirineo catalán. Por el contrario, en cuencas perturbadas, la exportación de sedimento puede alcanzar valores muy elevados y el sedimento en suspensión constituye una mayor proporción que los solutos respecto al total.

Entre los sólidos disueltos, $\mathrm{HCO}_{3}^{-}$y $\mathrm{Ca}^{2+}$ representan más del $50 \%$ de la salida total de solutos, debido a las características del sustrato rocoso. Esto es consistente con otros estu- 
dios en cuencas con predominio de rocas calcáreas, donde $\mathrm{HCO}_{3}^{-}$puede alcanzar el $64 \%$ del total de solutos, mientras $\mathrm{Ca}^{2+}$ representa alrededor del $25 \%$ (LLORENS y GALLART, 1992). De acuerdo con WALLING y WEBB (1996) el calcio $\left(\mathrm{Ca}^{2+}\right)$ y el bicarbonato $\left(\mathrm{HCO}_{3}{ }^{-}\right)$ representan los iones dominantes en la mayor parte de las aguas del mundo (véase también CRABTREE y TRUDGILL, 1984). Pero uno de los resultados más destacados de este trabajo es que $\mathrm{HCO}_{3}^{-}$y $\mathrm{Ca}^{2+}$ aumentan mucho proporcionalmente en la escorrentía de los usos del suelo menos exportadores de sedimento, demostrando así que los nutrientes más importantes del suelo $\left(\mathrm{Mg}^{2+}, \mathrm{K}^{+}, \mathrm{Na}^{+}, \mathrm{NO}_{3}^{-}\right)$están bien controlados por una densa cubierta de matorral o por los prados. Este no es el caso de los usos del suelo con mayot erosión (cultivo de cereal, barbecho, agricultura nómada), en los que la proporción de $\mathrm{HCO}_{3}^{-}$y $\mathrm{Ca}^{2+}$ disminuye en la escorrentía superficial, mientras aumentan los restantes nutrientes.

Una comparación entre la entrada de nutrientes con el agua de lluvia y la salida con el agua de escorrentía superficial muestra que para algunos nutrientes $\left(\mathrm{Ca}^{2+}, \mathrm{K}\right)$ las salidas superan a las entradas. Por el contrario, en el caso de otros nutrientes $\left(\mathrm{N}, \mathrm{Na}^{+}\right.$y $\mathrm{Mg}^{2+}$ ) el suelo actúa aparentemente como un sumidero, incorporando más cantidades desde la atmósfera de lo que se exporta con la escorrentía superficial. La información disponible para $P$ no es suficiente para establecer ninguna tendencia. No obstante, cualquier intento de balance de nutrientes a escala de ladera o de cuenca es imposible de realizar sin tener en cuanta los sólidos disueltos en la escorrentía subsuperficial y subterránea.

La conclusión final es que los usos del suelo que representan las mayores perturbaciones de suelo y cubierta vegetal son las fuentes más importante de solutos. Tales usos están también sujetos a la pérdida de los nutrientes más limitantes, confirmando así la importancia de la cubierta vegetal y de los procesos bióticos en la regulación de la pérdidas de solutos (SWANK, 1986). BEKE et al. (1989) llegaron a conclusiones similares al estudiar las características del sedimento en relación con las prácticas de cultivo y laboreo (véase también LAL, 1996). El abandono agrícola en laderas pendientes y la colonización por el matorral de antiguos campos significa un descenso en la salida de solutos, restringida principalmente a los iones $\left(\mathrm{HCO}_{3}^{-}\right.$y $\left.\mathrm{Ca}_{2}{ }^{+}\right)$más abundantes.

\section{AGRADECIMIENTOS}

Este trabajo se ha elaborado con el apoyo de los siguientes proyectos de investigación: «Water resources management in a changing environment: The impact of sediment on sustainability» - WARMICE (ENV4-CT98-0789) financiado por la Comisión Europea y "Producción de sedimentos y escorrentía como consecuencia de los cambios de uso del suelo en áreas de montaña» (AMB 96-0401) y «Estaciones permanentes para el estudio de procesos hidrológicos en ambientes mediterráneos» (HID98-1056-C02-01), ambos financiados por la CICYT. Los autores agradecen al Dr. Melchor Maestro sus sugerencias para mejorar el trabajo. 


\section{BIBLIOGRAFÍA}

ALVERA, B. (1990): Ciclos de elementos minernles en dos pinares pirenaicos. Unpubl. Thesis, Universidad Complutense de Madrid, 754, Madrid.

BEKE, G.J., LINDWALL, C.W., ENTZ, T. y CHANNAPPA, T.C. (1989): «Sediment and runoff water characteristics as influenced by cropping and tillage practices». Canadian Journal of Soil Science, 69: 639-647.

CRABTREE, R.W. y TRUDGILL, S.T. (1984): «Hydrochemical budgets for a Magnesium Limestone catchment in lowland England». Journal of Hydrology, 74, 67-79.

EVANS, R. (1995): «Some methods of directly assessing water erosion of cultivated land. A comparison of measurements made on plots and in fields». Progress in Physical Geography, 19(1), 115-130.

FELLER, M.C. y KIMMINS, J.P. (1984): «Effects of clearcutting on streamwater chemistry and watershed nutrient budgets in southern British Columbia». Water Resources Research, 20, 29-40.

GARCÍA-RUIZ, J.M., LASANTA, T., ORTIGOSA, L., RUIZ-FLAÑO, P., MARTÍ, C. y GONZÁLEZ, C. (1995): «Sediment yield under different land-uses in the Spanish Pyrenees». Mountain Research and Development, 15(3), 229-240.

GARCÍA-RUIZ, J.M., PUIGDEFÁBREGAS, J. y CREUS, J. (1986): «La acumulación de nieve en el Pirineo Central y su influencia hidrológica». Pirineos, 127, 27-72.

GARCÍA-RUIZ, J.M., ALVERA, B., Del BARRIO, G. y PUIGDEFÁBREGAS, J. (1990): «Geomorphic processes above timberline in the Spanish Pyrenees». Mountain Research and Development, 10 (3), 201-214.

GARCÍA-RUIZ, J.M., LASANTA, T., GONZÁLEZ, C., MARTÍ, C., WHITE, S., ERREA, M.P. y MAESTRO, M. (1996): «La agricultura marginal como fuente de sedimentos en el Pirineo Central». Cuadernos Lab. Xeol. Laxe, 21, 123-132.

IMESON, A.C. y VIS, M. (1984): «Seasonal variations in soil erodibility under different land-use types in Luxembourg». J. Soil Science, 35, 323-334.

KOSMAS, C., DANALATOS, N., CAMMERAAT, L.H., CHABART, M., DIAMANTOPOULOS, J., FARAND, L., GUTIÉRREZ, L., JACOB, A., MARQUÉS, H., MARTÍNEZ-FERNÁNDEZ, J., MIZARA, A., MOUSTAKAS, N., NICOLAU, J.M., OLIVEROS, C., PINNA. C., PUDDU, R., PUIGDEFÁBREGAS, J., ROXO, M., SIMAO, A., STAMOU, G., TOMASI, N., USAI, D. y VACCA, A. (1997): «The effect of land use on runoff and soil erosion rates under Mediterranean conditions». Catena, 29 (1), 45-60.

LAL, R. (1996): «Deforestation and land-use effects on soil degradation and rehabilitation in Western Nigeria. III. Runoff, soil erosion and nutrient loss». Land Degradation and Development, 7(2), 99-120.

LASANTA, T. (1988): «The process of desertion of cultivated areas in the Central Spanish Pyrenees». Pirineos, 132, 15-36. 
LASANTA, T. (1989): Evolución reciente de la agricultura de montaña: El Pirineo aragonés. Geoforma Ediciones, 220 p., Logroño.

LÓPEZ BERMÚDEZ, F., GARCÍA RUIZ, J.M., ROMERO DÍAZ, M.A., RUIZ FLAÑO, P., MARTÍNEZ FERNÁNDEZ, J. y LASANTA, T. (1993): «Medidas de flujos de agua y sedimentos en parcelas experimentales». Cuadernos Técnicos de la $S E G, 6,38$, Logroño.

LLORENS, P. y GALLART, F. (1992): «Small basin response in a Mediterranean mountainous abandoned farming area: Research design and preliminary results». Catena, 19(3-4), 309-320.

LLORENS, P., QYERALT, I., PLANA, F. y GALLART, F. (1997): «Studying solute and particulate sediment transfer in a small Mediterranean mountainous catchment subject to land abandonment». Earth Surface Processes and Landforms, 22; 1027-1035.

MOLINILLO, M., LASANTA, T. y GARCÍA-RUIZ, J.M. (1997): «Managing mountainous degraded landscapes after farmland abandonment in the Central Pyrenees». Environmental Management, 21(4), 587-598.

PAPY, F. y BOIFFIN, J. (1989): «The use of farming systems for the control of runoff and erosion». Soil Technology, 1, 29-39.

PARDINI, G., ARINGHIERI, R., PLANA, F. y GALLART, F. (1991): «Soil properties relevant to land degradation in abandoned sloping fields in Aísa Valley, Central Pyrenees, Spain». Pirineos, 137, 79-93.

PIONKE, H.B. y De WALLE, D.R. (1994): «Streamflow generation on a small agricultural catchment during autumn discharge: I. Non stormflow periods». Journal of Hydrology, 163, 1-22.

PUIGDEFÁBREGAS, J. y ALVERA, B. (1986): «Particulate and dissolved matter in snowmelt runoff from small watersheds». Z. Geomorph., 58, 69-80.

RIJCKBORST, H. (1967): «Hydrology of the upper-Garonne basin». Leidse Geologische Mededelingen, $40,1-74$.

RUIZ-FLAÑO, P. (1993): Procesos de erosión en campos abandonados del Pirineo. Geoforma Ediciones, 220 p., Logroño.

RÜTTIMAN, M., SCHAUB, D., PRASHUN, V. y RÜEGG, W. (1995): «Measurement of runoff and soil erosion on regularly cultivated fields in Switzerland». Catena, 25 (1-4), 127-140.

SMALING, E.M.A. y OENEMA, O. (1997): «Estimating nutrient balances in agro-ecosystems at different spatial scales». In Methods for assessment of soil degradation (R. LAL, W.H. BLUM, C. VALENTINE y B.A. STEWART, eds.), CRC Press, 229-252, Boca Raton.

SWANK, W.T. (1986): «Biological control of solute losses from forest ecosystems». In Solute processes (S.T. TRUDGILL, ed.), J. Wiley, 85-139, Chichester.

TRUDGILL, S.T. (1986): «Introduction». In Solute processes (S.T. TRUDGILL, ed.), J. Wiley, 1-14, Chichester. 
VERSTRATEN, J.M. (1977): «Chemical erosion in a forested watershed in the Oesling, Luxembourg». Earth Surface Processes, 2, 175-184.

WALLING, D.E. y Webb, B.W. (1986): «Solutes in river systems». In Solute processes (S. T. TRUDGILL, ed.), Wiley, , 251-327,Chichester. 